\title{
Dense Relativistic Electron Mirrors from a Laguerre-Gaussian Laser-Irradiated Micro-Droplet
}

\author{
Li-Xiang Hu ${ }^{1}$, Tong-Pu Yu ${ }^{1,2, *}$, Han-Zhen Li ${ }^{1}$, Yan Yin ${ }^{1}$, Paul McKenna ${ }^{2}$, and Fu-Qiu ShaO ${ }^{1}$ \\ ${ }^{1}$ Department of Physics, National University of Defense Technology, Changsha 410073, China \\ ${ }^{2}$ SUPA Department of Physics, University of Strathclyde, Glasgow G4 ONG, UK \\ *Corresponding author: tongpu@nudt.edu.cn
}

Compiled April 15, 2018

\begin{abstract}
We investigate dense relativistic electron mirror generation from a micro-droplet driven by circularlypolarized Laguerre-Gaussian lasers. The surface electrons are expelled from the droplet by the laser radial electric field and evolve into dense sheets after leaving the droplet. These electrons are trapped in the potential well of the laser transverse ponderomotive force and are steadily accelerated to about $100 \mathrm{MeV}$ by the inherent longitudinal electric field. Particle-in-cell simulations indicate that the relativistic electron mirrors are characterized by high beam charge, narrow energy spread and large angular-momentum, which can be utilized for bright compact $\mathrm{X} / \gamma$-ray pulse generation and photon vortex formation. (c) 2018 Optical Society of America
\end{abstract}

OCIS codes: (320.7090) Ultrafast lasers; (020.2649) Strong field laser physics; (350.5400) Plasmas; (350.4990) Particles.

http://dx.doi.org/10.1364/ao.XX.XXXXXX

Relativistic electron mirrors (REMs) can be used to generate compact $X / \gamma$-ray radiation sources required for fundamental and applied physics [1, 2], to study the Unruh and Hawking effects at the terrestrial laboratory conditions [3], or to achieve an electromagnetic wave intensification towards reaching the critical field of quantum electrodynamics [4,5]. The concept of the REM is firstly proposed by Einstein as a classical application of the Lorentz transformation formalism in the special theory of relativity [6]. In the concept, a counter-propagating electromagnetic wave is reflected by a relativistic flying mirror, and its frequency and amplitude increase by $4 \gamma_{M}^{2}$ assuming the reflection coefficient is equal to one, where $\gamma_{M}=\left(1-\beta_{x}^{2}\right)^{-0.5}$ is the mirror Lorentz factor and $\beta_{x}$ the normalized longitudinal electron velocity. At the same time, the duration of the incident pulse is compressed by $1 / 4 \gamma_{M}^{2}$. This ideal model is first demonstrated experimentally by the interaction of a high-intensity laser pulse with a freestanding, nm-thin foil [7]. However, the generated REMs disperse quickly before being adequately accelerated due to outward transverse ponderomotive force of the driving Gaussian pulse and the charge Coulomb repulsive effect [8]. To solve this problem, various laser-plasma based schemes are proposed. For example, Wu et al. added an additional foil to reflect the drive laser pulse and suppress the transverse momentum of the
REMs [9]. Stupakov et al. proposed to use a tailored laser pulse to simultaneously focus and accelerate REMs via the laser ponderomotive force [10]. In Esirkepov's concept of double-sided mirror, a dense thin slab accelerated in the radiation pressure regime by one laser pulse was employed to reflect another relativistically strong electromagnetic wave [11]. However, the produced mirrors via these schemes using solid foils are limited to either low $\gamma_{M}$ value or short lifetime. Alternatively, Bulanov et al. and Li et al. considered a parabolic REM generation in a plasma wakefield driven by intense electromagnetic fields [5, 12]. Meanwhile, Liseykina et al. proposed to generate relativistic attosecond electron bunches from droplets illuminated by a normal Gaussian laser pulse. However, the produced bunches have a large emission angle, low beam density and unstable structure, making the bunches inadequately suitable for REMs [13]. In our previous research [14], we found that a circularly-polarized Laguerre-Gaussian (LG) laser pulse with special beam profile can be used to trap electrons efficiently. This may provide us with a novel way to suppress the beam divergence and generate REMs with high $\gamma_{M}$, large beam charge and density.

In this Letter, we present an all-optical method to generate dense REMs with high $\gamma_{M}$ value and sub-nC beam charge. A circularly-polarized LG laser pulse with mode $(1,0)$ and a micro-droplet are employed. When the laser pulse interacts with the droplet, annular electron bunches are expelled from the droplet by the radial component of the laser electric field. Once leaving from the droplet, these electron bunches gradually evolve into periodically-distributed dense electron sheets, and are tightly trapped within the potential well of the laser transverse ponderomotive force, instead of being scattered by the laser pulse as reported previously $[8,13,15]$. At the same time, the trapped electrons are effectively accelerated by the cylindrically-symmetric longitudinal laser electric field and potentially show quasi-monoenergetic energy spectrum together with large angular-momentum (AM). This makes the REMs attractive for ultra-bright short $\mathrm{X} / \gamma$-ray pulse train emission and photon vortex formation via nonlinear Thomson scattering.

A series of simulations are carried out to investigate the dynamic process of the generation of dense REMs from laserdroplet interactions via the full 3D particle-in-cell (PIC) code Virtual Laser Plasma Lab (VLPL [16]). The size of the simulation box is $24 \lambda_{0} \times 12 \lambda_{0} \times 12 \lambda_{0}(x \times y \times z)$, with 50 grids per wavelength in all directions and 27 macro-particles in each 
cell for both electrons and ions, where $\lambda_{0}=1 \mu \mathrm{m}$ is the laser wavelength. The time resolution is set to $\Delta t=0.01 T_{0}$, where $T_{0}=3.3 \mathrm{fs}$ denotes the laser cycle. Absorbing boundary conditions for the fields and particles are employed in the propagation direction, while periodic ones for the fields and absorbing ones for the particles are used in the $y, z$ directions. Here we make use of a pre-ionized, cold liquid-helium droplet with a diameter of $D=2 \lambda_{0}$, which is centered at $x=4 \lambda_{0}$. The initial electron and ion density are respectively $n_{e}=10 n_{c}$ and $n_{H e^{2+}}=5 n_{c}$, where $n_{c}=1.12 \times 10^{21} \mathrm{~cm}^{-3}$ is the critical density for a $\lambda_{0}=1 \mu \mathrm{m}$ laser. The collisions between particles are neglected in the simulations. A circularly-polarized LG laser pulse with mode $(1,0)$ is employed, and it is focused at $x=3 \lambda_{0}$ and propagates along the $x$-axis. The laser electric field $\vec{E}\left(L G_{0}^{1}\right)$ is given by $\vec{E}_{\perp}\left(L G_{0}^{1}\right)=\sqrt{2 e} E_{L 0} \rho \sigma_{0} \sigma^{-2} \exp \left(-\rho^{2} / \sigma^{2}\right) L_{0}^{1}\left(2 \rho^{2} / \sigma^{2}\right) G(x-$ ct) $\left(\sin \xi \vec{e}_{y}-\cos \xi \vec{e}_{z}\right)$, where $E_{L 0}=m_{e 0} c \omega_{0} a_{0} / q_{e}$ is the peak amplitude of the laser electric field with $m_{e 0}$ the electron rest mass, $c$ the speed of light, $\omega_{0}$ the laser frequency, $q_{e}$ the fundamental charge, and $a_{0}=10$ the laser dimensionless vector potential, $\rho=\left(y^{2}+z^{2}\right)^{1 / 2}, \sigma=\sigma_{0}\left(1+x^{2} / f^{2}\right)^{1 / 2}$ with $\sigma_{0}=3 \lambda_{0}$ the focal spot radius, $x$ the distance from the beam waist, and $f=\pi \sigma_{0}^{2} / \lambda_{0}$ the Rayleigh length, $G(x-c t)=$ $\cos ^{2}[\pi(x-c t) /(2 c \tau)]$ for $-c \tau \leq x-c t \leq c \tau$ with $\tau=5 T_{0}$, and $L_{0}^{1}\left(2 \rho^{2} / \sigma^{2}\right)$ is the generalized Laguerre polynomial. Here, $\xi=-k x+\omega_{0} t-k r^{2} /(2 R)-2 \arctan (x / f)+\varphi$ denotes the phase term with $k$ the wave number, $R=x+f^{2} / x$ the curvature radius of the wave-front, $2 \arctan (x / f)$ the Guoy phase of the mode, and $\varphi$ the azimuthal angle. Such an intense LG-mode laser pulse can be generated via a spiral-shaped foil [17], plasma holograms [18] or stimulated Raman scattering.

When the LG laser pulse interacts with the helium droplet, annular electron bunches are expelled from the target every laser cycle and propagate along the $x$-axis, as is shown in Fig. 1(a). During a normal Gaussian laser pulse interacting with a microdroplet, atto-second electron bunches are either emitted from the droplet surface in the laser polarization plane every halflaser cycle with certain angle with respect to the laser axis [13], or are continuously swept from the droplet and form a spiral structure [19]. The distinction in our scheme can be attributed to the unique field structure of the laser pulse. A circularlypolarized LG laser pulse consists of two mutually perpendicular linearly-polarized components with a $\pi / 2$ phase displacement. For convenience, we convert the laser transverse electric field $E_{y}$ and $E_{z}$ into the radial and azimuthal components, owing to $E_{\rho}=\cos \varphi \cdot E_{y}+\sin \varphi \cdot E_{z}$ and $E_{\varphi}=-\sin \varphi \cdot E_{y}+\cos \varphi$. $E_{z}$, namely $E_{\rho}=\sqrt{2 e} E_{L 0} \rho \sigma_{0} \sigma^{-2} \exp \left(-\rho^{2} / \sigma^{2}\right) G(x-c t) \sin \xi^{\prime}$, and $E_{\varphi}=-\sqrt{2 e} E_{L 0} \rho \sigma_{0} \sigma^{-2} \exp \left(-\rho^{2} / \sigma^{2}\right) G(x-c t) \cos \xi^{\prime}$, where $\xi^{\prime}=$ $k\left(x+\rho^{2} / 2 R\right)-\omega t-2 \arctan [(x-c t) / f]$ is the transformed phase term. One will see that $E_{\rho}$ is circularly-symmetric with respect to the $x$-axis. The circular symmetry of the field configuration causes circularly-symmetric electron dynamics, which is of critical importance for the annular electron bunch generation. The above equations also imply that $E_{\rho}$ is periodical in the $x$ axis with a spacing of $1 \lambda_{0}$. When it is positive $\left(-q_{e} E_{\rho}<0\right)$, the electrons in the skin layer of the micro-droplet are compressed inside, forming a dense electron sheath; as it becomes negative $\left(-q_{e} E_{\rho}>0\right)$, the electrons feel radially outward tensile force and are dragged out of the target, so that an annular thin electron mirror forms. Since the cycle of $E_{\rho}$ along the $x$ direction is exactly $1 T_{0}$, these mirrors are emitted every laser cycle. By analyzing the field structure, we can also explain well the sawtooth-like/spiral
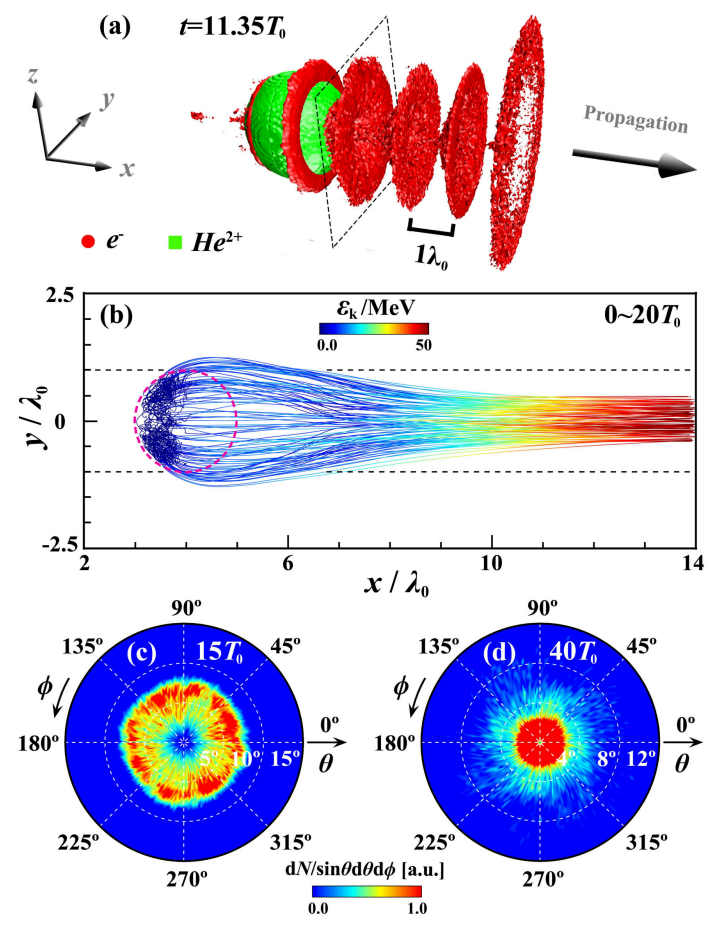

Fig. 1. Electron and ion density isocontour surfaces corresponding to $0.5 n_{c}$ at $11.35 T_{0}(\mathrm{a})$. The projection of electron trajectories in the $x y$ plane until $20 T_{0}(b)$, and the red circle denotes the droplet profile. Electron emission angle versus the azimuthal angle at $15 T_{0}(\mathrm{c})$ and $40 T_{0}(\mathrm{~d})$. Here, $\theta=\arctan \left(p_{\perp} / p_{x}\right), \phi=\arctan (z / y)$, and $p_{\perp}=\left(p_{y}^{2}+p_{z}^{2}\right)^{1 / 2}$.

electron bunch formation as reported elsewhere [13, 19].

Usually when the laser with $(0,0)$ mode sweeps the droplet surface, electrons are dragged out with an emission angle with respect to the laser propagation direction. Due to the outward transverse ponderomotive force and the Coulomb repulsive effect, the electron bunches quickly dissolve in the laser fields, just like ripples spreading in water. However, the stable structure of the electron mirrors is robust in the case introduced here. After escaping from the micro-droplet, the electrons in each mirror converge into an array of discs, as is shown in Fig. 1(a) and (b), and continue being accelerated. However, ions expand in-situ slowly due to the larger mass-to-charge ratio. Fig. 1(c) shows the distribution of electron emission angle $\theta$ versus the azimuthal angle $\phi$ at $t=15 T_{0}$. It's inferred that $\theta$ ranges from $3^{\circ}$ to $8^{\circ}$. Without any external force, the mirror would rapidly expand in space, accompanied by a sharp density decrease. Fortunately, these mirrors obtained via our scheme are trapped in the potential well of the inward laser transverse ponderomotive force and the emission angle of electrons steeply descends to $\theta<3^{\circ}$ at $t=40 T_{0}$, as is shown in Fig. 1(d). The mirror structure remains intact for a long time without obvious dispersion in space, as seen in Fig. 2(a). Moreover, the average electron density decreases only from $3 n_{c}$ to $1.5 n_{c}$ over $20 T_{0}$, while the beam diameter changes roughly from $0.8 \lambda_{0}$ to $1.2 \lambda_{0}$ accordingly, as indicated in Fig. 2(b) and (c). Comparing with other schemes $[5,9,13]$, the produced REMs here are either much denser and stabler or with much higher beam charge, and may have larger mirror reflectivity.

We can qualitatively interpret the REM confinement in the transverse direction using the ponderomotive potential model $[20,21]$. Here, the time-averaged force experienced by an 
electron is given by $F_{\text {pond }}=-\nabla V_{\text {pond }}(\rho, x, t)=-m_{e} c^{2} \nabla[(1+$ $\left.\left.|a(\rho, x, t)|^{2} / 2\right)^{1 / 2}-1\right]$, where $V_{\text {pond }}(\rho, x, t)$ is the ponderomotive potential and $a(\rho, x, t)$ is the normalized amplitude of the laser electric field. For the azimuthal component of $F_{\text {pond }}$ is zero, we get the transverse ponderomotive force:

$$
F_{\rho}=\left(\frac{2 \rho^{2}}{\sigma^{2}}-1\right) \frac{\sqrt{2 e} q_{e} E_{L 0} \sigma_{0}}{k \sigma^{2}} \exp \left(-\rho^{2} / \sigma^{2}\right) G(x-c t) .
$$

It's inferred from Eq. (1) that when $\rho>\sqrt{2} \sigma / 2$, the transverse pondermotive force $F_{\rho}$ is positive, which pushes electrons outward radially, and the situation reverses in the inverse range. In order to trap the REMs, these electrons have to stay within the potential well with $\rho<\sqrt{2} \sigma / 2$ (where $F_{\rho}<0$ ). As shown in Fig. 2(b) and (c), most electrons are trapped in the red dashed circles with radius of $0.4-0.6 \lambda_{0}$, which are located exactly within the potential well. Thus, the droplet radius plays a key role in electron trapping. Once the radius exceeds $\sqrt{2} \sigma / 2$, the bunches are not trapped by $F_{\rho}$ any more. On the other hand, the laser misalignment is also an important factor in experiments. However, our additional simulations show that the same physics occurs for a droplet target with a transverse offset up to $0.5 \lambda_{0}$.

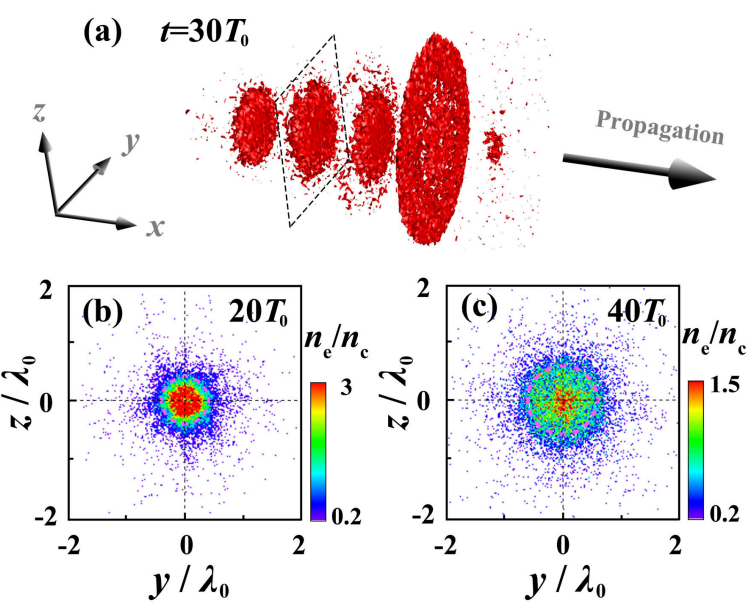

Fig. 2. Electron density isocontour surfaces corresponding to $0.5 n_{c}$ at $30 T_{0}(\mathrm{a})$. Transverse density distribution of an electron bunch marked in the panel (a) at $20 T_{0}$ (b) and $40 T_{0}$ (c) and the corresponding red circle radii are $0.4 \lambda_{0}$ and $0.6 \lambda_{0}$, respectively.

Although the potential well of the inward transverse ponderomotive force plays a key role in trapping the REMs, the space charge effect also plays an important part in determining the beam charge and divergence in space. Here the transverse Coulomb field can be estimated as $E_{\perp}^{\mathcal{S}} \approx\left(4 \pi \epsilon_{0}\right)^{-1}\left(q_{e} N \gamma / \sigma_{0}^{2}\right)$, where $\epsilon_{0}$ is the permittivity, and $N$ the electron number. Considering the relativistic cancellation of the magnetic and electric forces, the net transverse repulsion force approximates to $f_{\perp}^{s} \approx\left(4 \pi \epsilon_{0}\right)^{-1} q_{e}^{2} N /\left(\gamma \sigma_{0}^{2}\right)$. Since the net transverse repulsion force is in proportion to the reciprocal of the electron Lorentz factor, i.e., $f_{\perp}^{s} \sim 1 / \gamma$, the net transverse repulsion force can be neglected $\left(f_{\perp}^{\bar{s}}\right.$ ultimately approaches 0$)$ for electrons with $\gamma \gg 2$. Actually, the simulation results in Fig. 3 show that the peak electron energy at $t=40 T_{0}$ is above $100 \mathrm{MeV}(\gamma \simeq 200)$. For the electron bunch marked in Fig. 2(a), it's estimated that the transverse ponderomotive force is four orders of magnitude larger than the net transverse repulsion force. Thus the influence of the space charge effect on each REM can be reasonably ignored as compared to the transverse ponderomotive force in Eq. (1).
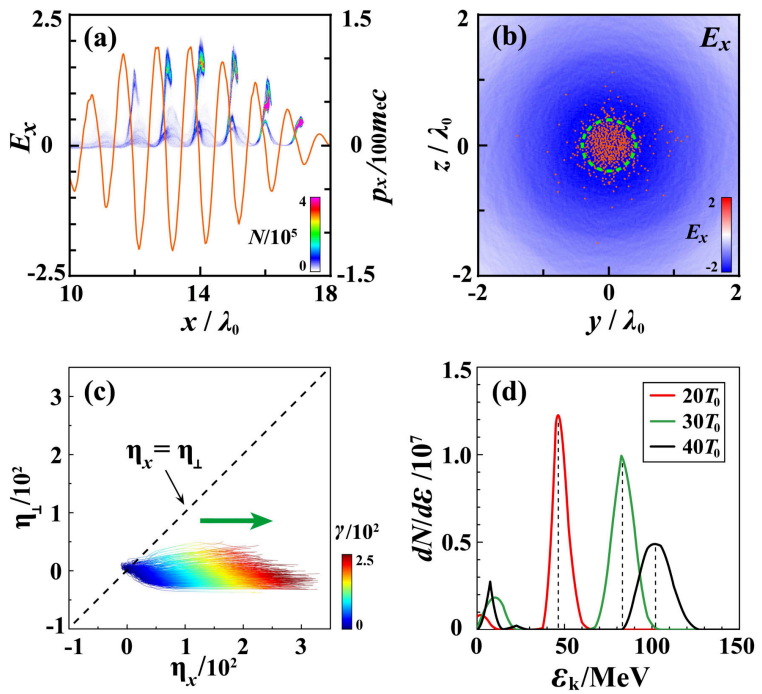

Fig. 3. Distribution of the electrons in $\left(x, p_{x}\right)$ space at $20 T_{0}$ and the corresponding axial electric field $E_{x}(\mathrm{a})$. Transverse distribution of the laser longitudinal electric field $E_{x}$ experienced by an electron bunch marked in Fig. 2 at 20T, with red dots representing the location and a green circle the profile (b). Trajectories of the tracked electrons in the two orthogonal axes of the energy gain $\left(\eta_{x}, \eta_{\perp}\right)$ space until $60 T_{0}$ and the green arrow denotes the electron acceleration direction (c). The energy spectra of the electron bunch (d) as indicated in Fig. 2(a). The electric fields here are normalized by $E_{0}=m_{e} c \omega_{0} / q_{e}$.

After escaping from the droplet, the electron mirrors continue being accelerated by the laser field. The laser longitudinal electric field is usually self-generated or converted from a normal $(0,0)$ mode in plasmas $[22,23]$, while the situation changes for a circularly-polarized LG laser pulse. The expression of the existing longitudinal electric field can be obtained from the Poisson equation, $E_{x}(\rho, \varphi, x)=-(i / k)\left(\partial E_{y} / \partial y+\partial E_{z} / \partial z\right)$ :

$$
E_{x} \simeq\left(\frac{\rho^{2}}{\sigma^{2}}-1\right) \frac{2 \sqrt{2 e} E_{L 0} \sigma_{0}}{k \sigma^{2}} \exp \left(-\rho^{2} / \sigma^{2}\right) G(x-c t) \cos \xi^{\prime} .
$$

It's obvious that the on-axis longitudinal electric field is periodic along the $x$-axis. When the on-axis $E_{x}<0\left(-q_{e} E_{x}>0\right)$, electrons are accelerated forward, and the situation reverses when $E_{x}>0\left(-q_{e} E_{x}<0\right)$. It's shown that the bunch electrons are exactly located in the acceleration phase of the LG laser fields in Fig. 3(a). In the transverse cross-section with $x=c t$, $E_{x} \sim-2 \sqrt{2 e} E_{L 0} /\left(k \sigma_{0}\right)$ at the center axis and vanishes at $\rho=\sigma_{0}$; For $\rho<\sigma_{0}, E_{x}<0\left(-q_{e} E_{x}>0\right)$, which is able to accelerate electrons forward; For $\rho>\sigma_{0}, E_{x}>0\left(-q_{e} E_{x}<0\right)$, electrons are decelerated. It's shown that the electrons located in a green circle with respect to the $x$-axis with a $0.4 \lambda_{0}$ radius are exactly within the acceleration region in Fig. 3(b). Moreover, one sees that the field $E_{x}$ is isotropic in the transverse cross-section, as its expression in Eq. (2) is the same for different azimuthal angle $\varphi$, resulting in isotropic acceleration for each electron mirror.

In order to explore the detailed dynamics, we track some typical electrons and present their energy evolution in Fig. 3(c). Here we separate the two driving terms in the energy equation $m_{e 0} c^{2} d \gamma / d t=-q_{e}\left(E_{x} v_{x}+\vec{E}_{\perp} \cdot \vec{v}_{\perp}\right)$ with $\vec{E}_{\perp} \cdot \vec{v}_{\perp}=E_{y} v_{y}+E_{z} v_{z}$, where $v_{x}, v_{y}, v_{z}$ are the three components of the electron velocity. Thus the energy gains due to the longitudinal and transverse electric fields are $\eta_{x}=-\frac{q_{e}}{m_{e 0} c^{2}} \int_{0}^{t} E_{x} v_{x} d t$ and $\eta_{\perp}=$ 

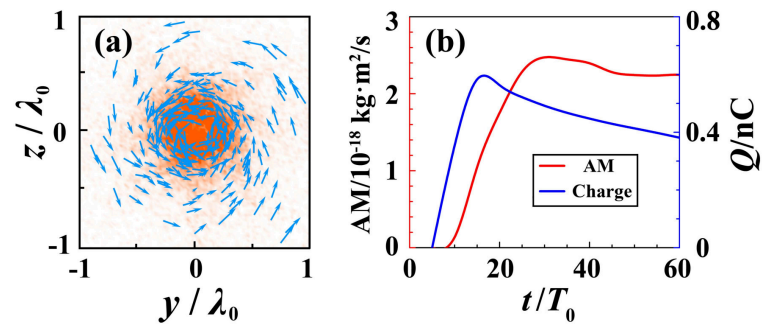

Fig. 4. Electron velocity field with AM larger than $6.8 \times$ $10^{-29} \mathrm{~kg} \cdot \mathrm{m}^{2} / \mathrm{s}$ in the $y z$ plane with $x=14 \lambda_{0}$ at $20 T_{0}$ (a). Temporal evolution of the electron AM and beam charge (b).

$-\frac{q_{e}}{m_{e 0} c^{2}} \int_{0}^{t} \vec{E}_{\perp} \cdot \vec{v}_{\perp} d t$, respectively. As expected, the contribution of the transverse electric fields is smaller and almost all electrons are accelerated along the $\eta_{x}$ direction, indicating the dominance of longitudinal electric field acceleration over transverse acceleration. For $0 \leq \rho \leq 0.5 \lambda_{0},\left|E_{x}\right| /\left|E_{x}\right|_{\text {max }}$ only changes from 1 to 0.94 , indicating quasi-monoenergetic acceleration of each bunch. The final electron energy can be approximately estimated as $\varepsilon_{k, \text { max }}=e \bar{E}_{x} L_{a}$, where $L_{a}$ is the acceleration length. Actually, for the bunches in our scheme, $\gamma_{M} \approx \gamma \approx 100$, i.e., $\gamma_{M} / \gamma \simeq 1$, leading to an extremely high Doppler shift factor $4 \gamma_{M}^{2} \simeq 4 \gamma^{2}$, which is much higher than that in the double-sided mirror concept using a high-intensity laser pulse [11]. This makes the flying mirrors very promising for generating brilliant femto/atto-second $\mathrm{X} / \gamma$-ray pulses. For example, brilliant $\gamma$-photon bursts with a total photon number above $10^{9}(>1 \mathrm{MeV})$ in each burst can be obtained, when we increase the laser intensity to $a_{0}=30$.

For a circularly-polarized LG laser pulse, both its spin and orbital AM can be transferred to electrons and cause electron rotation [24-26]. Fig. 4(a) presents the velocity field of electrons with AM larger than $6.8 \times 10^{-29} \mathrm{~kg} \cdot \mathrm{m}^{2} / \mathrm{s}$ in the $y z$ plane. Most electrons rotate around the origin point and form a vortex. In a cylindrical coordinate system, the average change rate of the electron AM per unit volume is governed by[25]

$$
m_{e} n_{e} \rho \frac{d v_{\varphi}}{d t}=-q_{e} n_{e} \rho\left(E_{\varphi}+v_{x} B_{\rho}-v_{\rho} B_{x}\right)-\frac{d M_{x}}{d t}
$$

where $n_{e}$ is the electron number density, $v_{\rho, \varphi, x}$ the electron velocity, and $M_{x}$ the axis component of the AM density. Eq. (3) shows that the electromagnetic field $E_{\varphi}, B_{\rho}$, or $B_{x}$ and the AM absorption $d M_{x} / d t$ provide the dissipation mechanism for the AM transfer. The total electron AM calculated is shown in Fig. 4(b), where $\mathcal{L}=\sum_{i=1}^{n}\left|\mathbf{r}_{\mathbf{i}} \times \mathbf{p}_{\mathbf{i}}\right|=\sum_{i=1}^{n}\left[\left(y_{i}-y_{0}\right) p_{z, i}-\left(z_{i}-z_{0}\right) p_{y, i}\right]$ with $y_{0}, z_{0}$ the axis position in the $y z$ plane. At $t=60 T_{0}$, $\mathcal{L} \sim 10^{-18} \mathrm{~kg} \cdot \mathrm{m}^{2} / \mathrm{s}$, which may be useful for electron microscopy and other applications [27]. Compared with the mirrors from thin foils [9], this unique property makes the bunches more attractive for the generation of bright $X / \gamma$-ray vortices, via nonlinear Thompson scattering or synchrotron radiation [28].

In conclusion, we report on an efficient scheme for generating stable and dense relativistic electron mirrors from a circularlypolarized Laguerre-Gaussian laser-irradiated micro-droplet. The surface electrons expelled from the droplet by the laser radial electric field evolve into dense mirrors and are trapped efficiently in the potential well of the laser transverse ponderomotive force. The mirrors are characterized by high beam charge, narrow energy spread, low beam divergence, and large AM, which can be utilized for bright femto/atto-second $X / \gamma$-ray pulse train generation and photon vortex formation.
Funding. NSFC (Grant Nos. 11622547, 11675264, 11474360, 11475030), Science Challenge Project (Grant No. TZ2016005), Hunan Provincial Natural Science Foundation of China (Grant No. 2017JJ1003) and Hunan Provincial Science and Technology Program (Grant No. 2017RS3042), Fok Ying-Tong Education Foundation (Grant No. 161007), Research Project of NUDT (JQ14-0202), and EPSRC (Grant No. EP/M018091/1 and EP/R006202/1).

\section{REFERENCES}

1. G. A. Mourou, T. Tajima, and S. V. Bulanov, Rev. Mod. Phys. 78, 309371 (2006).

2. M. Kando, Y. Fukuda, A. S. Pirozhkov, J. Ma, I. Daito, L.-M. Chen, T. Zh. Esirkepov, K. Ogura, T. Homma, Y. Hayashi, H. Kotaki, A. Sagisaka, M. Mori, J. K. Koga, H. Daido, S. V. Bulanov, T. Kimura, Y. Kato, and T. Tajima, Phys. Rev. Lett. 99, 135001 (2007).

3. P. Chen and G. Mourou, Phys. Rev. Lett. 118, 045001 (2017).

4. S. V. Bulanov, T. Zh. Esirkepov, M. Kando, and J. Koga, Plasma Source Sci. Technol. 25, 053001 (2016).

5. S. V. Bulanov, T. Esirkepov, and T. Tajima, Phys. Rev. Lett. 91, 085001 (2003).

6. A. Einstein, Ann. Phys. Leipz. 17, 891 (1905).

7. D. Kiefer, M. Yeung, T. Dzelzainis, P. S. Foster, S. G. Rykovanov, C. L. S. Lewis, R. S. Marjoribanks, H. Ruhl, D. Habs, J. Schreiber, M. Zepf, and B. Dromey, Nat. Commun. 4, 1763 (2013).

8. V. V. Kulagin, V. A. Cherepenin, M. S. Hur, and H. Suk, Phys. Rev. Lett. 99, 124801 (2007).

9. H. C. Wu, J. Meyer-ter-Vehn, J. Fernández, and B. M. Hegelich, Phys. Rev. Lett. 104, 234801 (2010).

10. G. V. Stupakov and M. S. Zolotorev, Phys. Rev. Lett. 86, 5274-5277 (2001).

11. T. Zh. Esirkepov, S. V. Bulanov, M. Kando, A. S. Pirozhkov, and A. G. Zhidkov, Phys. Rev. Lett. 103, 025002 (2009).

12. F. Y. Li, Z. M. Sheng, Y. Liu, J. Meyer-ter-Vehn, W. B. Mori, W. Lu, and J. Zhang, Phys. Rev. Lett. 110, 135002 (2013).

13. T. V. Liseykina, S. Pirner, and D. Bauer, Phys. Rev. Lett. 104, 095002 (2010).

14. L. X. Hu, T. P. Yu, Z. M. Sheng, J. Vieira, D. B. Zou, Y. Yin, P. McKenna, and F. Q. Shao, Sci. Rep. (submitted).

15. L. X. Hu, T. P. Yu, F. Q. Shao, D. B. Zou, and Y. Yin, Phys. Plasmas 22, 033104 (2015).

16. A. Pukhov, J. Plasma Phys. 61, 425-433 (1999).

17. Y. Shi, B. F. Shen, L. G. Zhang, X. M. Zhang, W. P. Wang, and Z. Z. Xu, Phys. Rev. Lett. 112, 235001 (2014).

18. A. Leblanc, A. Denoeud, L. Chopineau, G. Mennerat, Ph. Martin, and F. Quéré, Nature Phys. 13, 440-443 (2017).

19. T. P. Yu, L. X. Hu, Y. Yin, F. Q. Shao, H. B. Zhuo, Y. Y. Ma, X. H. Yang, W. Luo, and A. Pukhov, Appl. Phys. Lett. 105, 114101 (2014).

20. B. Quesnel and P. Mora, Phys. Rev. E 58, 3719-3732 (1998).

21. Q. Kong, S. Miyazaki, S. Kawata, K. Miyauchi, K. Sakai, Y. K. Ho, K. Nakajima, N. Miyanaga, J. Limpouch, and A. A. Andreev, Phys. Rev. E 69, 056502 (2004).

22. D. B. Zou, A. Pukhov, L. Q. Yi, H. B. Zhuo, T. P. Yu, Y. Yin, and F. Q. Shao, Sci. Rep. 7, 42666 (2017).

23. K. D. Xiao, T. W. Huang, L. B. Ju, R. Li, S. L. Yang, Y. C. Yang, S. Z. Wu, H. Zhang, B. Qiao, S. C. Ruan, C. T. Zhou, and X. T. He, Phys. Rev. E 93, 043207 (2016).

24. M. G. Haines, Phys. Rev. Lett. 87, 135005 (2001).

25. S. Ali, J. R. Davies, and J. T. Mendonca, Phys. Rev. Lett. 105, 035001 (2010).

26. L. B. Ju, C. T. Zou, T. W. Huang, K. Jiang, H. Zhang, S. Z. Wu, B. Qiao, and S. C. Ruan, Phys. Rev. E 95, 053205 (2017).

27. B. J. McMorran, A. Agrawal, I. M. Anderson, A. A. Herzing, H. J. Lezec, J. J. McClelland, and J. Unguris, Science 331, 192-195 (2011).

28. M. Katoh, M. Fujimoto, H. Kawaguchi, K. Tsuchiya, K. Ohmi, T. Kaneyasu, Y. Taira, M. Hosaka, A. Mochihashi, and Y. Takashima, Phys. Rev. Lett. 118, 094801 (2017). 


\section{REFERENCES}

1. G. A. Mourou, T. Tajima, and S. V. Bulanov, "Optics in the relativistic regime," Rev. Mod. Phys. 78, 309-371 (2006).

2. M. Kando, Y. Fukuda, A. S. Pirozhkov, J. Ma, I. Daito, L.-M. Chen, T. Zh. Esirkepov, K. Ogura, T. Homma, Y. Hayashi, H. Kotaki, A. Sagisaka, M. Mori, J. K. Koga, H. Daido, S. V. Bulanov, T. Kimura, Y. Kato, and T. Tajima, "Demonstration of Laser-Frequency Upshift by Electron-Density Modulations in a Plasma Wakefield," Phys. Rev. Lett. 99, 135001 (2007).

3. P. Chen and G. Mourou, "Accelerating Plasma Mirrors to Investigate the Black Hole Information Loss Paradox," Phys. Rev. Lett. 118, 045001 (2017).

4. S. V. Bulanov, T. Zh. Esirkepov, M. Kando, and J. Koga, "Relativistic mirrors in laser plasmas (analytical methods)," Plasma Source Sci. Technol. 25, 053001 (2016).

5. S. V. Bulanov, T. Esirkepov, and T. Tajima, "Light Intensification towards the Schwinger Limit," Phys. Rev. Lett. 91, 085001 (2003).

6. A. Einstein, "On the Electrodynamics of Moving Bodies," Ann. Phys. Leipz. 17, 891 (1905).

7. D. Kiefer, M. Yeung, T. Dzelzainis, P. S. Foster, S. G. Rykovanov, C. L. S. Lewis, R. S. Marjoribanks, H. Ruhl, D. Habs, J. Schreiber, M. Zepf, and B. Dromey, "Relativistic electron mirrors from nanoscale foils for coherent frequency upshift to the extreme ultraviolet," Nat. Commun. 4, 1763 (2013).

8. V. V. Kulagin, V. A. Cherepenin, M. S. Hur, and H. Suk, "Theoretical Investigation of Controlled Generation of a Dense Attosecond Relativistic Electron Bunch from the Interaction of an Ultrashort Laser Pulse with a Nanofilm," Phys. Rev. Lett. 99, 124801 (2007).

9. H. C. Wu, J. Meyer-ter-Vehn, J. Fernández, and B. M. Hegelich, "Uniform Laser-Driven Relativistic Electron Layer for Coherent Thomson Scattering," Phys. Rev. Lett. 104, 234801 (2010).

10. G. V. Stupakov and M. S. Zolotorev, "Ponderomotive laser acceleration and focusing in vacuum for generation of attosecond electron bunches," Phys. Rev. Lett. 86, 5274-5277 (2001).

11. T. Zh. Esirkepov, S. V. Bulanov, M. Kando, A. S. Pirozhkov, and A. G. Zhidkov, "Boosted High-Harmonics Pulse from a Double-Sided Relativistic Mirror," Phys. Rev. Lett. 103, 025002 (2009).

12. F. Y. Li, Z. M. Sheng, Y. Liu, J. Meyer-ter-Vehn, W. B. Mori, W. Lu, and J. Zhang, "Dense Attosecond Electron Sheets from Laser Wakefields Using an Up-Ramp Density Transition," Phys. Rev. Lett. 110, 135002 (2013).

13. T. V. Liseykina, S. Pirner, and D. Bauer, "Relativistic Attosecond Electron Bunches from Laser-Illuminated Droplets," Phys. Rev. Lett. 104, 095002 (2010).

14. L. X. Hu, T. P. Yu, Z. M. Sheng, J. Vieira, D. B. Zou, Y. Yin, P. McKenna, and F. Q. Shao, "Attosecond electron bunches from a nanofiber driven by Laguerre-Gaussian laser pulses," Sci. Rep. (submitted).

15. L. X. Hu, T. P. Yu, F. Q. Shao, D. B. Zou, and Y. Yin, "Enhanced dense attosecond electron bunch generation by irradiating an intense laser," Phys. Plasmas 22, 033104 (2015).

16. A. Pukhov, "Three-dimensional electromagnetic relativistic particlein-cell code VLPL (Virtual Laser Plasma Lab)," J. Plasma Phys. 61, 425-433 (1999).

17. Y. Shi, B. F. Shen, L. G. Zhang, X. M. Zhang, W. P. Wang, and Z. Z. Xu, "Light Fan Driven by a Relativistic Laser Pulse," Phys. Rev. Lett. 112, 235001 (2014).

18. A. Leblanc, A. Denoeud, L. Chopineau, G. Mennerat, Ph. Martin, and F. Quéré, "Plasma holograms for ultrahigh-intensity optics," Nature Phys. 13, 440-443 (2017)

19. T. P. Yu, L. X. Hu, Y. Yin, F. Q. Shao, H. B. Zhuo, Y. Y. Ma, X. H. Yang, W. Luo, and A. Pukhov, "Bright tunable femtosecond $\mathrm{x}$-ray emission from laser irradiated micro-droplets," Appl. Phys. Lett. 105, 114101 (2014).

20. B. Quesnel and P. Mora, "Theory and simulation of the interaction of ultraintense laser pulses with electrons in vacuum," Phys. Rev. E 58, 3719-3732 (1998)

21. Q. Kong, S. Miyazaki, S. Kawata, K. Miyauchi, K. Sakai, Y. K. Ho, K. Nakajima, N. Miyanaga, J. Limpouch, and A. A. Andreev, "Electron bunch trapping and compression by an intense focused pulse laser," Phys. Rev. E 69, 056502 (2004).

22. D. B. Zou, A. Pukhov, L. Q. Yi, H. B. Zhuo, T. P. Yu, Y. Yin, and F. Q. Shao, "Laser-Driven Ion Acceleration from Plasma Micro-Channel Targets," Sci. Rep. 7, 42666 (2017).

23. K. D. Xiao, T. W. Huang, L. B. Ju, R. Li, S. L. Yang, Y. C. Yang, S. Z. Wu, H. Zhang, B. Qiao, S. C. Ruan, C. T. Zhou, and X. T. He, "Energetic electron-bunch generation in a phase-locked longitudinal laser electric field," Phys. Rev. E 93, 043207 (2016).

24. M. G. Haines, "Generation of an Axial Magnetic Field from Photon Spin," Phys. Rev. Lett. 87, 135005 (2001).

25. S. Ali, J. R. Davies, and J. T. Mendonca, "Inverse Faraday Effect with Linearly Polarized Laser Pulses," Phys. Rev. Lett. 105, 035001 (2010).

26. L. B. Ju, C. T. Zou, T. W. Huang, K. Jiang, H. Zhang, S. Z. Wu, B. Qiao, and S. C. Ruan, "Production of high-angular-momentum electron beams in laser-plasma interactions," Phys. Rev. E 95, 053205 (2017).

27. B. J. McMorran, A. Agrawal, I. M. Anderson, A. A. Herzing, H. J. Lezec, J. J. McClelland, and J. Unguris, "Electron Vortex Beams with High Quanta of Orbital Angular Momentum," Science 331, 192-195 (2011).

28. M. Katoh, M. Fujimoto, H. Kawaguchi, K. Tsuchiya, K. Ohmi, T. Kaneyasu, Y. Taira, M. Hosaka, A. Mochihashi, and Y. Takashima, "Angular Momentum of Twisted Radiation from an Electron in Spiral Motion," Phys. Rev. Lett. 118, 094801 (2017). 